\title{
Reconstruction of Inferior Right Hepatic Veins in Living Donor Liver Transplantation
}

\author{
NICOLAE BACALBASA ${ }^{1,2}$, IRINA BALESCU ${ }^{3}$, MIHAI PAUTOV $^{4}$, JULIAN BREZEAN ${ }^{1,5^{*}}$, \\ MIHAELA VILCU ${ }^{1,5^{*}}$ and VLADISLAV BRASOVEANU 4 \\ ${ }^{1}$ Carol Davila University of Medicine and Pharmacy, Bucharest, Romania; \\ ${ }^{2}$ Center of Excellence in Translational Medicine, Fundeni Clinical Institute, Bucharest, Romania; \\ ${ }^{3}$ Ponderas Academic Hospital, Bucharest, Romania; \\ ${ }^{4}$ Dan Setlacec Center of Gastrointestinal Disease and Liver Transplantation, \\ Fundeni Clinical Institute, Bucharest, Romania; \\ 5 “I. Cantacuzino” Clinical Hospital, Bucharest, Romania
}

\begin{abstract}
Background: A proper knowledge of the anatomy of the liver (including its vascular particularities) is mandatory in cases which are going to be submitted to major hepatic resection, including living donor liver transplantation. Case Report: We present the case of a living donor liver transplantation in which a particularity of the anatomy of the hepatic veins was reported for the donor: two inferior hepatic veins for segments 5 and 6. This particularity imposed the need for creation of a supplemental anastomosis in the recipient: a phleboplasty of the two inferior veins followed by direct re-implantation into the inferior cava vein. However, the postoperative course was uneventful for both the donor and the recipient. Conclusion: In certain cases presenting vascular particularities such as two inferior hepatic veins, phleboplasty followed by reimplantation into the inferior cava vein might be needed in order to provide a good vascular outflow of the liver graft.
\end{abstract}

Living donor liver transplantation has been widely implemented in order to provide a faster solution for patients with end-stage liver disease and reduce the waiting time, most often right lobe grafts being used in order to minimize the small-for-size liver syndrome. However, appropriate

This article is freely accessible online.

*These Authors contributed equally to this study.

Correspondence to: Irina Balescu, 2A Caraiman Street, Buzau, Romania. Tel: +40 724077709, e-mail: irina.balescu@ ponderasah.ro

Key Words: Inferior right hepatic vein, phleboplasty, living donor liver transplantation. knowledge of the particularities of the venous drainage is mandatory in order to avoid hepatic venous congestion and its complications (consisting of early graft dysfunction or even sepsis) $(1,2)$. Reported for the first time by Yamaoka et al. in 1994 (3), the right liver graft is currently preferentially performed in certain centers, one of the most important steps of the procedure consisting of achieving an appropriate outflow reconstruction $(3,4)$.

Therefore, the anatomical details of the retrohepatic inferior cava vein, as well as of the hepatic veins ostium play a central role in cases proposed for major liver resection including liver transplantation in order to achieve a good functional outcome for both the donor and the recipient (5).

We present the case of a living donor liver transplantation in which a particularity of the anatomy of the hepatic veins was reported for the donor, necessitating a change in surgical approach.

\section{Case Report}

The recipient of the liver was a 51-year-old female diagnosed with two hepatocarcinomatous lesions in segments IV and VII, developed on liver cirrhosis due to chronic viral hepatitis (B and D) with portal and parenchymatous deterioration. The patient had a Model for End-Stage Liver Disease (MELD) score of 13 and two episodes of upper digestive hemorrhage in the previous year, successfully treated in a minimally conservative manner.

The donor was her son, 27 years of age, with no known medical history. In terms of liver volumetry, the donor had a total hepatic volume of $1777 \mathrm{~cm}^{3}$ and a right hepatic volume of $1,155 \mathrm{~cm}^{3}$ (representing the donated hepatic mass and $65 \%$ of the entire liver volume). The left lobe had a volume of $622 \mathrm{~cm}^{3}$ (representing $35 \%$ of the entire liver parenchyma); the graft weight/recipient weight ratio was 1.8 . 
In terms of vascularization, the portal vein presented a modal left and right distribution, while the hepatic artery for segments 2 and 3 originated from the left gastric artery. The donor was found to have an arterial branch for segment 4 from the right hepatic artery. In terms of parenchymatous venous outflow, the donor presented two accessory veins of 5 and $6 \mathrm{~mm}$ for segments 5-6 and 6, while the biliary tree presented no particular distribution (Figure 1).

The two patients were submitted to surgery, a right hemiliver transplantation from living related donor being performed; the recipient was submitted to the following anastomoses: the right hepatic vein of the donor was sutured through an end-to-side anastomosis with the inferior cava vein; therefore, a phleboplasty of the two inferior hepatic veins and re-implantation into the inferior cava vein through end-to-side anastomosis was performed (Figures 2, 3A and B). The right portal vein of the donor was sutured to the right portal vein of the recipient by an end-to-end anastomosis, the right hepatic artery of the donor was sutured to the right hepatic artery of the recipient through an end-to-end anastomosis while the right hepatic bile duct of the donor was sutured through an end-to-end anastomosis with the common hepatic bile duct of the recipient, (Figures 4 and 5).

The postoperative outcome of the both patients was uneventful, the ultrasound examination which was performed in the next postoperative day showed normal flow in all the vascular structures.

\section{Discussion}

In the past decade, implementation of vascular surgery techniques in visceral surgery have led to a wider performance of extended visceral resections involving major vascular structures (6-9). Experience achieved in treating such cases was further successfully applied in liver transplantation from both living and cadaveric donors $(10,11)$.

However, a proper knowledge of the anatomy of the hepatic veins is mandatory in patients who will be submitted to liver transplantation, especially for the recipient, in order to establish which type of vascular reconstruction is feasible. Moreover, data reported so far in literature regarding the vascular anatomy of the liver are usually inconsistent, an important fact leading to these modifications being the patient's race; therefore, whenever vascular surgery is needed, a proper knowledge of the anatomical particularities of each patient is mandatory $(12,13)$.

The classical notions of anatomy describe the right hepatic vein as a vascular structure originating from antero-inferior angle of the liver, running in the intersectional plane between the right anterior and right posterior sections of the liver, ensuring the outflow of the segments 6 and 7 and, to a degree, the outflow of segments 5 and 8. In the eighth segment, the right hepatic vein lies horizontally and it enters

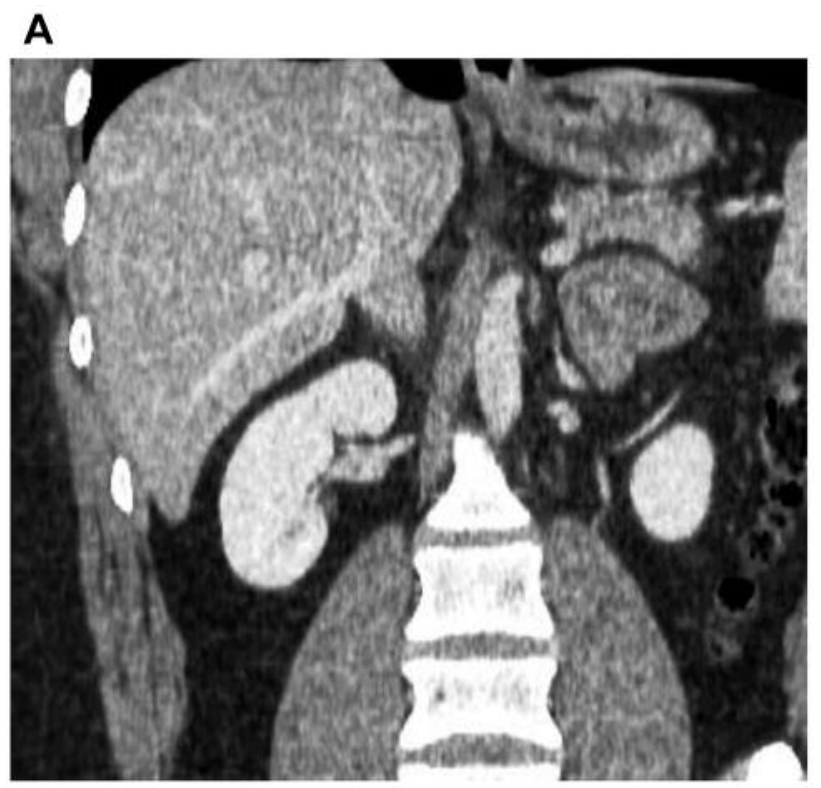

B

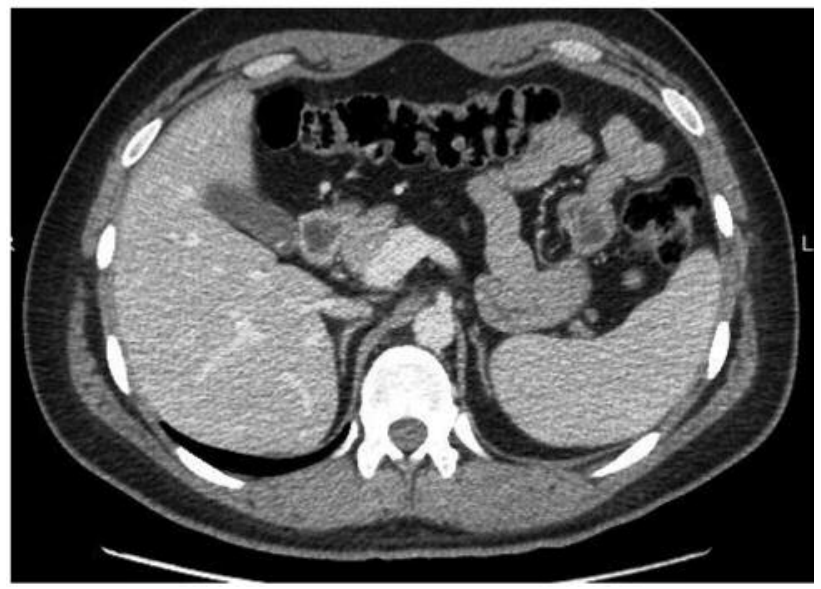

Figure 1. Preoperative computed tomography of the donor. A: Sagittal section revealing the presence of an inferior right hepatic vein. B: Transverse section revealing the presence of an inferior right hepatic vein.

the inferior cava vein a few millimeters lower than the common trunk of the middle and left hepatic vein. In certain cases, accessory inferior right hepatic veins draining directly into the inferior cava vein have been reported (14).

Joshi et al. studied the anatomy of the inferior cava vein as well as the anatomy of the hepatic veins in 69 cadavers (13). The authors reported a median length of the intrahepatic cava vein of $5.7 \mathrm{~cm}$ and a median diameter of $2.3 \mathrm{~cm}$, while in terms of anatomy of the hepatic veins, the authors reported a normal anatomy (consisting of a right hepatic vein and a common trunk between left and middle hepatic vein) in $65 \%$ of cases. Among cases presenting inferior hepatic veins, the 


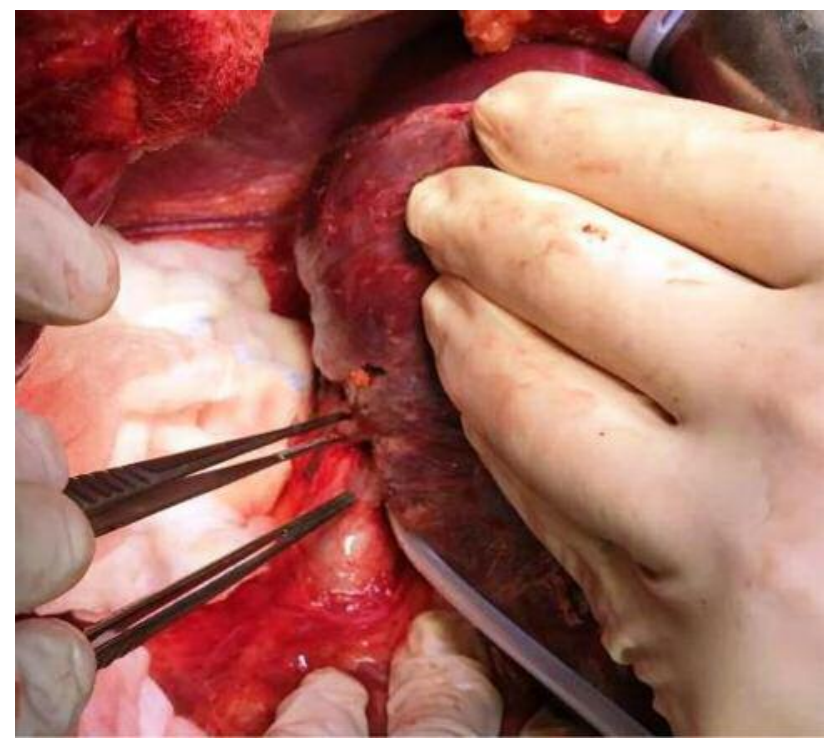

Figure 2. Intraoperative aspect after mobilization of the right hemi-liver, identification of the two inferior right hepatic veins.

reported number of such structures ranged between two and 16 branches (with a median of seven veins) (13).

Another study regarding the anatomy of the inferior cava vein, as well as the ostia of the hepatic veins, was conducted by Ghosh et al. (5). The study was conducted on 160 cadaveric adult human liver specimens in which the distribution of the hepatic venous ostia was studied after dividing the retrohepatic area of the inferior cava vein into 16 quadrants. The diameter of the hepatic ostia was classified as large when it exceeded $1 \mathrm{~cm}$, medium when between 0.5 and $1 \mathrm{~cm}$, small when between 0.1 and $0.5 \mathrm{~cm}$ and very small then less than $0.1 \mathrm{~cm}$. Moreover, the large openings were further classified as superior large openings (located in the upper four quadrants) and inferior large openings (located in the lower four quadrants). According to the same study, the right postero-lateral vein and the right postero-superior vein were defined according to their rapport with the right postero-inferior vein in terms of intracaval drainage. According to this study, the median length of the retohepatic cava vein was $7.3 \mathrm{~cm}$ (range 6.2-8.4 cm), while the median diameter of the openings of the hepatic ostia was $2 \mathrm{~cm}$ for the right ostium, $1.8 \mathrm{~cm}$ for the left ostium and $1.7 \mathrm{~cm}$ for the inferior ostium. In the meantime, the median number of hepatic venous openings per case was seven (range 5-9). The ostium for the right inferior hepatic vein was single in 78 cases while in the remaining cases double, triple or even quadruple openings were reported. According to the diameter of the opening, this was classified as large in $42.3 \%$ of cases, medium in $56.4 \%$ of cases and small in $1.3 \%$ of cases. When it comes to the distribution of the ostia

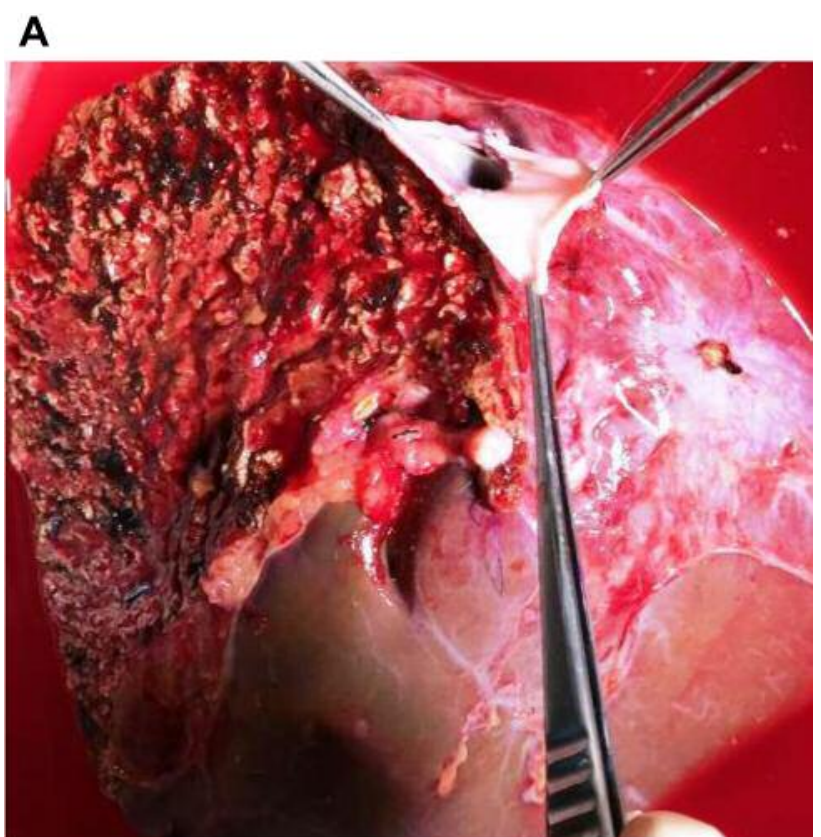

B

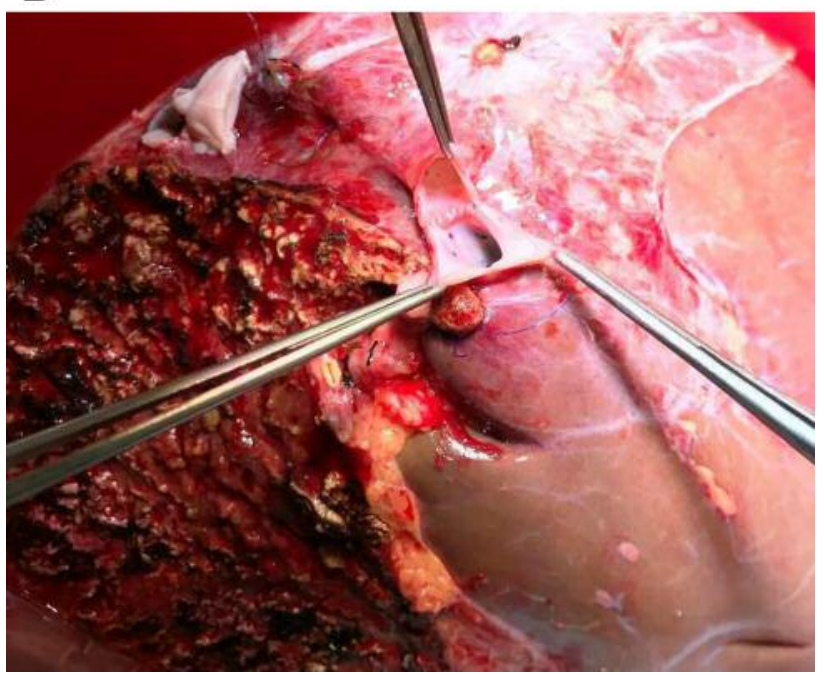

Figure 3. A: Preparation of the right hepatic vein. B: Venoplasty of the two inferior right hepatic veins.

on the surface of the retrohepatic inferior cava vein, the authors underlined the fact that these openings are situated preferentially on the right hemi-diameter of the inferior cava vein, this finding being extremely important especially during performing a hanging maneuver of the liver as well as for patients considered in order to donate the right hemiliver (6). A similar finding to those reported in Ghosh et al.'s study was seen in our case; the donor presented two accessory inferior right hepatic veins, each vein presenting its own ostium on the right surface of the retrohepatic 


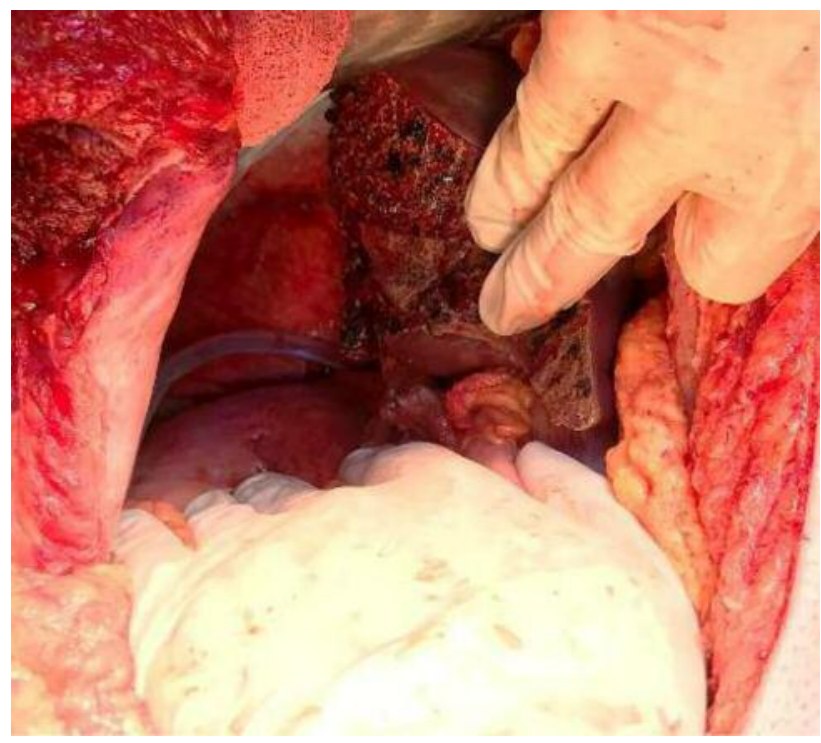

Figure 4. Final aspect of the donor after right hepatectomy.

inferior cava vein. Due to the fact that in our case the diameter of the ostiums were quite small $(5$ and $6 \mathrm{~mm}$ respectively), a prior phleboplasty of the two venous structures was mandatory before implantation into the inferior cava vein. Therefore, a proper knowledge of the possible anatomical particularities in such cases is needed in order to achieve a good functional outcome.

A report on a similar case to ours was published by Turkish surgeons in 2015 (2). Similar to our case, the recipient of the liver was a 56-year-old female with previous history of autoimmune hepatitis-induced liver failure, while the donor was her 30-year-old son. The preoperative workup conducted on the donor demonstrated a right lobe liver volume of $991 \mathrm{~cm}^{3}$ and a remnant liver volume of $729 \mathrm{~cm}^{3}$, as well as a classical distribution of the portal vein and proper hepatic artery; however, an anatomic particularity of the hepatic veins was observed: the patient presented a right hepatic vein with a diameter of $14 \mathrm{~mm}$, a second hepatic vein besides the right hepatic vein measuring $13 \mathrm{~mm}$ and draining segment 8 , and the last one, measuring $12 \mathrm{~mm}$, draining segment 6 . The anterior and the posterior right biliary branches opened separately in the main biliary duct. When inserting the graft, three out of the four hepatic veins were anastomosed with the recipient remnant venous orifices, while the fourth vein was reinserted in the inferior cava vein using a $10 \mathrm{~mm}$ polytetrafluoroethylene graft. The two biliary branches were anastomosed with the recipient's main hepatic bile duct. Both patients had an eventful postoperative evolution so they were discharged on the tenth postoperative day (2).

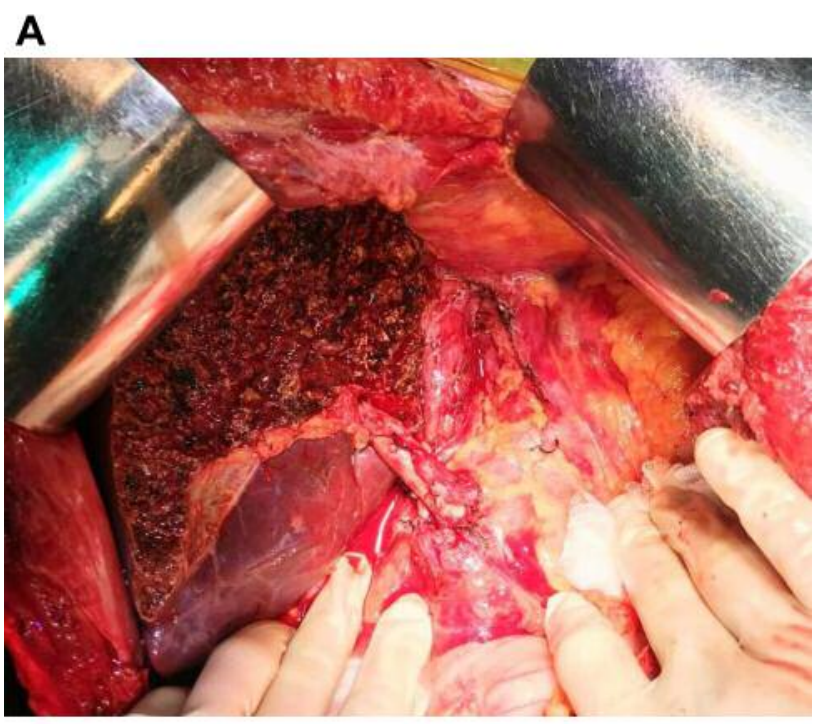

B

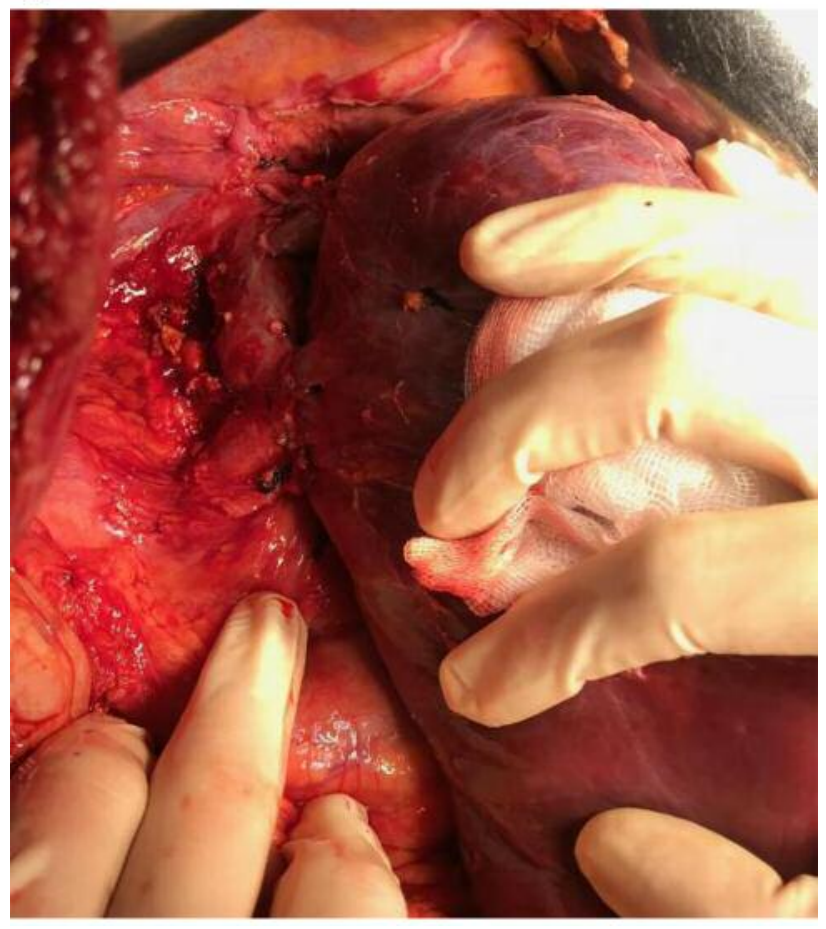

Figure 5. The final aspect of the recipient A: after placing the graft and $B:$ after re-implantation of the right hepatic vein and of the two inferior right hepatic veins into the inferior cava vein.

An important issue which should be taken into consideration whenever multiple inferior right hepatic veins are found in the liver graft is the risk of stenosis. This is why Huang et al. suggested that whenever multiple inferior right hepatic veins are found, unification venoplasty should be performed in order to minimize the risk of stenosis at the site 
of re-implantation into the inferior cava vein (15). In the study by this surgical team between July 2004 and February 2010 , the rate of stenosis in the group with single vein reinsertion was $23 \%$, while the same percentage decreased to $18.9 \%$ when venoplasty was associated; moreover, this value decreased to $2.9 \%$ once the procedure of funneling unification venoplasty was introduced (15).

A more recent study conducted on the theme of hepatic venous reconstruction in right liver living donor transplantation conducted by Ito et al. also underlined the importance of re-implantation of the inferior right hepatic vein into the inferior cava vein; the authors demonstrated that whenever an obstruction of the right hepatic veins occurs, a functional inferior right hepatic vein will provide a proper drainage route, improving in this way the functional reserve of the graft (16).

\section{Conclusion}

Although right liver living donor liver transplantation has been widely implemented in the past three decades, a proper knowledge of the anatomical particularities of both donor and recipient is mandatory in order to achieve a good functional result of the graft. The presence of one or more inferior right hepatic veins should be carefully identified preoperatively; once these anatomic particularities are observed, a direct re-implantation of the inferior veins in the inferior cava vein through venoplasty should be performed in order to achieve a better outflow of the graft.

\section{References}

1 Lee S, Park K, Hwang S, Lee Y, Choi D, Kim K, Koh K, Han S, Choi K, Hwang K, Makuuchi M, Sugawara Y and Min P: Congestion of right liver graft in living donor liver transplantation. Transplantation 71: 812-814, 2001.

2 Aktas S, Sevmis S, Ozgur S, Erol C and Karakayali H: Four separate hepatic vein reconstructions in living-donor right-lobe liver transplantation: Case Report. Transplant Proc 47: 30203022, 2015.

3 Yamaoka Y, Washida M, Honda K, Tanaka K, Mori K, Shimahara Y, Okamoto S, Ueda M, Hayashi M and Tanaka A: Liver transplantation using a right lobe graft from a living related donor. Transplantation 57: 1127-1130, 1994.

4 Sugawara Y, Makuuchi M, Akamatsu N, Kishi Y, Niiya T, Kaneko J, Imamura $\mathrm{H}$ and Kokudo N: Refinement of venous reconstruction using cryopreserved veins in right liver grafts. Liver Transpl 10: 541-547, 2004.

5 Ghosh SK and Paul S: Anatomy of the retrohepatic segment of the inferior vena cava and the ostia venae hepaticae with its clinical significance. Surg Radiol Anat 34: 347-355, 2012.
6 Bacalbasa N, Brezean I, Anghel C, Barbu I, Pautov M, Balescu $\mathrm{I}$ and Brasoveanu V: Management of a fulminant upper gastrointestinal bleeding exteriorized through hemobilia due to arteriobiliary fistula between the common bile duct and a right hepatic artery aneurysm - a case report. In Vivo 31: 983-989, 2017.

7 Bacalbasa N, Brezean I, Anghel C, Barbu I, Pautov M, Balescu $\mathrm{I}$ and Brasoveanu V: Successful resection and vascular ligation of a large hepatic artery aneurysm - a case report and literature review. In Vivo 31: 979-982, 2017.

8 Brasoveanu V, Anghel C, Barbu I, Pautov M, Ionescu MI, Motthor M, Balescu I, Dima S and Bacalbasa $\mathrm{N}$ : Pancreatoduodenectomy en bloc with portal and superior mesenteric artery resection - a case report and literature review. Anticancer Res 35: 1613-1618, 2015.

9 Pantea S, Jiga L, Ionac M and Lazar F: Arterio-venous fistula using nonpenetrating titanium clips (VCS). Chirurgia (Bucur) 108: 563-567, 2013.

10 Brasoveanu V, Anghel C, Bacalbasa N, Ionescu MI, Matei E, Barbu I, Grasu M, Tomescu D and Popescu I: Technical aspects of biliary reconstruction correlated with biliary complications in 46 consecutive right lobe liver transplantations from living donors. Chirurgia (Bucur) 109: 15-19, 2014.

11 Brasoveanu V, Ionescu MI, Grigorie R, Mihaila M, Bacalbasa N, Dumitru R, Herlea V, Iorgescu A, Tomescu D and Popescu I: Living donor liver transplantation for unresectable liver adenomatosis associated with congenital absence of portal vein: A case report and literature review. Am J Case Rep 16: 637-644, 2015.

12 Mehran R, Schneider R and Franchebois P: The minor hepatic veins: Anatomy and classification. Clin Anat 13: 416-421, 2000.

13 Joshi SD, Joshi SS and Siddiqui AU: Anatomy of retrohepatic segment of inferior vena cava and termination of hepatic veins. Indian J Gastroenterol 28: 216-220, 2009.

14 Lau SHY, Lai ECH and Lau WY: Anatomy in liver resection. In: Operative Techniques in Liver Resection. Yan L. (ed.). Springer, Dordrecht, 2016.

15 Hwang S, Ha TY, Ahn CS, Moon DB, Kim KH, Song GW, Jung DH, Park GC, Namgoong JM, Jung SW, Yoon SY, Sung KB, Ko GY, Cho B, Kim KW and Lee SG: Reconstruction of inferior right hepatic veins in living donor liver transplantation using right liver grafts. Liver Transpl 18: 238-247, 2012.

16 Ito K, Akamatsu N, Tani K, Ito D, Kaneko J, Arita J, Sakamoto Y, Hasegawa K and Kokudo N: Reconstruction of hepatic venous tributary in right liver living donor liver transplantation: The importance of the inferior right hepatic vein. Liver Transpl 22: 410-419, 2016. 